\title{
An Inter-disciplinary Modelling Approach in Industrial 5G/6G and Machine Learning Era
}

\author{
Abdelrahim Mohamed*, Hang Ruan*, Mohamed Heshmat Hassan Abdelwahab ${ }^{\dagger \S}$, Bogdan Dorneanu ${ }^{\ddagger}$, \\ Pei Xiao*, Harvey Arellano-Garcia ${ }^{\ddagger}$, Yang Gao ${ }^{\dagger}$, Rahim Tafazolli* \\ ${ }^{*}$ Institute for Communication Systems, Home of $5 \mathrm{GIC} \&{ }^{\dagger}$ Surrey Space Centre \& ${ }^{\ddagger}$ Chemical Process Engineering \\ University of Surrey, Guildford, U.K., GU2 7XH \\ ${ }^{\S}$ Department of Mathematics, Faculty of Science, Sohag University, Sohag, Egypt \\ Correspondence e-mail: abdelrahim.mohamed @ surrey.ac.uk
}

\begin{abstract}
Recently, the fifth-generation (5G) cellular system has been standardised. As opposed to legacy cellular systems geared towards broadband services, the $5 \mathrm{G}$ system identifies key use cases for ultra-reliable and low latency communications (URLLC) and massive machine-type communications (mMTC). These intrinsic 5G capabilities enable promising sensor-based vertical applications and services such as industrial process automation. The latter includes autonomous fault detection and prediction, optimised operations and proactive control. Such applications enable equipping industrial plants with a sixth sense (6S) for optimised operations and fault avoidance. In this direction, we introduce an inter-disciplinary approach integrating wireless sensor networks with machine learningenabled industrial plants to build a step towards developing this 6S technology. We develop a modular-based system that can be adapted to the vertical-specific elements. Without loss of generalisation, exemplary use cases are developed and presented including a fault detection/prediction scheme, and a sensor density-based boundary between orthogonal and non-orthogonal transmissions. The proposed schemes and modelling approach are implemented in a real chemical plant for testing purposes, and a high fault detection and prediction accuracy is achieved coupled with optimised sensor density analysis.
\end{abstract}

Index Terms-5G; big data; deep learning; machine learning; mMTC; network slicing; predictive analysis; URLLC.

\section{INTRODUCTION}

We, human beings, acquire information from our surroundings through our sensory receptors of vision, sound, smell, touch and taste -the five senses. The sensory stimulus is converted to electrical signals as nerve impulse data communicated with our brain. What is really intriguing is the communication network. When one or more senses fail (i.e., impairment), we are able to re-establish communication and improve our other senses to protect us from incoming dangers. Furthermore, we have developed the mechanism of "reasoning", effectively analyzing the present data and generating a vision of the future, which we might call our sixth sense $(6 S)$.

Is it possible to develop a $6 \mathrm{~S}$ technology to predict a catastrophic industrial disaster? Industrial processes are already equipped with five senses: "hearing" from acoustic sensors, "smelling" from gas and liquid sensors, "seeing" from camera, "touching" from vibration sensors and "tasting" from composition monitors. $6 \mathrm{~S}$ could be achieved by forming a sensing network which is self-adaptive and self-repairing, carrying out deep-thinking analysis with even limited data, and predicting the sequence of events via integrated system modelling. In this paper we introduce an inter-disciplinary modelling approach to develop a $6 \mathrm{~S}$ technology for industrial processes by bringing together components from beyond-4G and $5 \mathrm{G}$ wireless communications, machine learning, process systems, process control, robotics and autonomous systems. The 6S technology introduced in this paper could be further explored to a wide range of industrial and manufacturing processes.

In complex distributed sensory systems like industrial chemical plants, wireless sensor networks (WSN) often operate in potentially hostile and harsh environments in order to monitor or sense various aspects of the environments. In addition, these sensors are usually very sensitive and vulnerable to environmental conditions and can easily malfunction due to hardware failures or insecurely exposed to attacks and cause software failures [1]. Dysfunctional sensors can further affect the entire WSN by transmitting faulty data, occupying more network bandwidth inefficiently and increasing network power consumption, which can further lead to more severe consequences including inevitable damage to both of the cyber and physical systems and direct profit loss [1], [2]. Therefore, intelligent fault detection techniques are demanded to detect and identify faulty sensors and data in complex heterogeneous industrial systems.

In the practical scenarios of fault detection, much less faulty data are readily available compared to the amount of normal data. The data obtained from sensor collections and measurements is imbalanced if the classes are not approximately equally represented, in which case, the fault data represent only a small portion of ill process conditions. This prevents most fault detection algorithms being provided with a comprehensive and generalized knowledge of the fault types. In particular, when the class sizes are highly imbalanced, conventional classification algorithms tend to strongly favor the majority class and detect the minority class at extremely low rates [3].

Support vector machines (SVM), as one of standard conventional machine learning classification algorithms, has been adopted by the work in [3]-[6] to perform fault detection in WSN-assembled systems, due to its superior performance 
on moderate-sized high dimensional data. With a large feature range of the data, the faulty data can be easily separated between hyperplanes with either linear or nonlinear kernels specified by the algorithm, under the assumption that the normal and faulty data are balance distributed. Additionally, [4] provides several comparable machine learning techniques including K-nearest neighbors (KNN) and a Gaussian Mixture Model (GMM) to give better performance regarding sensitivity and accuracy in the application of chemical column leakage detection [4]. However, in more practical scenarios, it is insufficient to only detect the faults. This is because even the most sophisticated fault detection algorithms can only detect the faults only when they are already there (i.e., after the fault happens), which is meaningless sometimes, as damages and losses are already done and cannot not be reverted. This suggests fault prediction and predictive maintenance techniques. However, little research work has been done in this area. The authors of [7], [8] have discussed the general frameworks and designs of applying big data analytics with machine learning in internet-of-things (IoT) applications. In, [9] a deep belief network (DBN) is utilised to establish a predictive model that generates estimates and residuals for faulty signal detection and reconstructions. A novel deep learning approach is proposed in [10] to unfold nuclear power reactor signals with a combination of convolutional neural networks (CNN), de-noising auto-encoders (DAE) and kmeans clustering of representations.

In this paper, a compatible and integrated machine learning model is proposed in order to address both fault detection problems and fault prediction challenges at the same time in a $6 \mathrm{~S}$ system having a chemical process at the centre. This can be materialized as an online procedure by taking the high throughput and low latency advantages of $5 \mathrm{G}$ communication within the WSN. The contributions of this paper include: 1) We propose an inter-disciplinary system model built in a modular approach to achieve a $6 \mathrm{~S}$ technology. 2) We investigate requirements of the network slice required to achieve a certain WSN performance. 3) We propose a fault detection and prediction model based on machine learning. 4) We implement the proposed schemes in a real-world chemical plant including autonomous systems.

The reminder of this paper is structured as follows: Section II provides the system model and a high level overview of the main components. Section III introduces the wireless communication network and the statistical traffic model. Section IV develops the machine learning-based fault detection and prediction scheme. Section V provides an overview of the real-world testing plant. Section VI presents and discusses the results. Finally, conclusion are drawn in Section VII.

\section{Proposed System Architecture}

The proposed system architecture to enable the industrial $6 \mathrm{~S}$ consists of vertical-specific components/models overlaid by a $5 \mathrm{G}$ communication network. We focus on intelligent/smart process plants in chemical industries as a case study for the verticals. Fig. 1 shows a high level overview

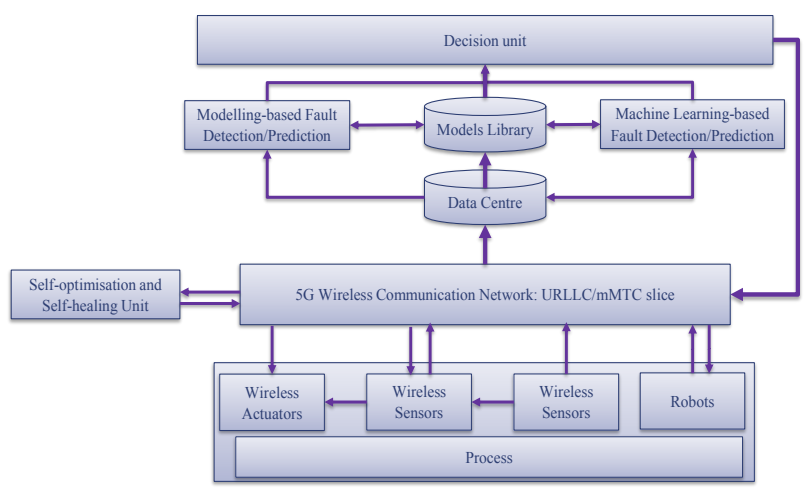

Figure 1. System Model: High level overview

of the proposed architecture. The architecture is designed in modular manner, and there are five main modules that constitute the envisioned structure. These include the holistic system models and cooperative control, massive connectivity and resilient network communication, machine learning-based fault detection and prediction, intelligent adaptive decisionmaking framework, and virtual reality system for visualization. The $6 \mathrm{~S}$ architecture divides to the physical layer and cyber layer. The former includes the industrial process itself, wireless sensors and actuators, the physical controllers, and the inspector robots. On the other hand, the cyber layer includes the wireless communication network, the data- and model-centres, fault detection/prediction algorithms, and the decision-making framework. The wireless network considers the connecting chain between the two layers.

Wireless sensors and actuators are deployed in the plant and connected to the cellular network. Robots are also deployed in the plant for periodic maintenance and surveillance as well as to act as relays in deep fading areas. All measurements are transmitted via the cellular network to a data centre. The latter is accessed by two different fault detection and prediction units. The machine learning unit performs fault detection and prediction based on the instantaneous and historical measurements. On the other hand, the modellingbased unit performs fault detection and prediction based on the models that characterise the underlying process. The results from both units are sent to the decision unit which analyses the signals and sends commands in the downlink to the wireless actuators via the cellular network. The detailed description and operation flow for these units are explained in the following sections.

\section{COMmunicAtion Network}

The legacy cellular systems have been designed primarily for human initiated mobile broadband communications. They are highly suboptimal for narrow band, short-bust, sporadic traffic (e.g., sensor measurement data, such as temperature, pressure, humidity, etc.) generated by sensors in chemical plants. It is envisioned that a new design paradigm is needed to support large numbers of heterogeneous sensing devices with diverse requirements and unique traffic characteristics. 


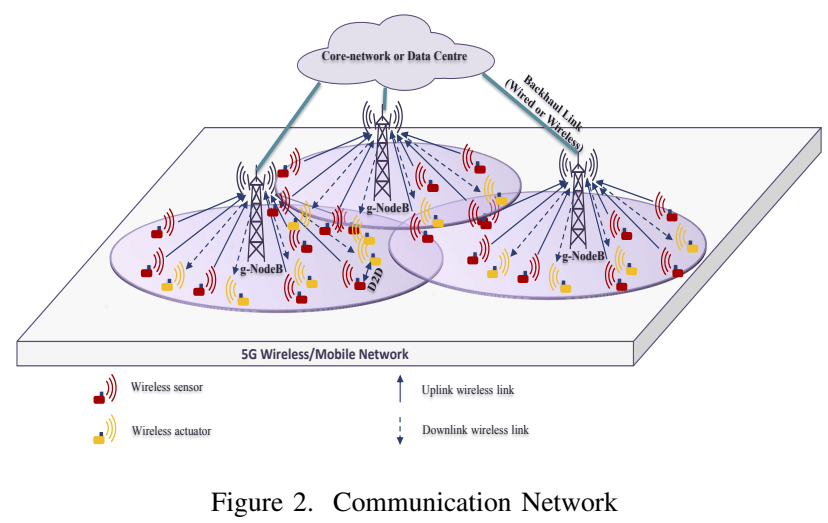

Comparing to the sensors in traditional IoT networks, those deployed in the extreme environments need to operate in harsh (sometimes hazardous) conditions, thus are prone to wear and tear, and cannot be easily replaced, posing major challenges in designing resilient networks for reliable communications.

We consider a centralised control mechanism where sensors are connected to a fusion node via wireless links as shown in Fig. 2. The wireless links can also be used to send commands to actuators within the plant. The network consists of a heterogeneous set of periodic and event-triggered sensors with mixed requirements, characteristics and traffic models. Considering heterogeneity of the plant and the associated sensors, a statistical model rather than a deterministic model is chosen for the sensor transmission events. The number of incoming packets (or events when each event generates a single packet) per unit of time follows the Poisson distribution while the packet interval is modelled as an exponential distribution. This results in probability-based transmissions that can be controlled by the arrival rate and the inter-arrival time.

In legacy systems, different users are allocated to orthogonal resources in the time/frequency/code domain in order to avoid or alleviate inter-user interference, thus they can be classified as orthogonal multiple access (OMA) techniques. The number of connected sensors/actuators is expected to grow in industrial processes, and will inevitably exceed the available resources, resulting in an overloaded condition, which may incur failed transmissions and network malfunction. In this direction, we define the boundary, in terms of sensor density, between conventional OMA and the candidate 5G technology, termed non-orthogonal multiple access (NOMA). This boundary is defined based on the maximum number of active sensors that can achieve a certain performance under pre-defined requirements. This can be expressed as:

$$
\begin{aligned}
& B=\max (D) \\
& \text { s.t. } L \leq 100 \%
\end{aligned}
$$

where $B$ is the boundary in terms of sensor/actuator density between OMA/NOMA, $D$ is the actual sensor/actuator density, and $L$ is the network load.

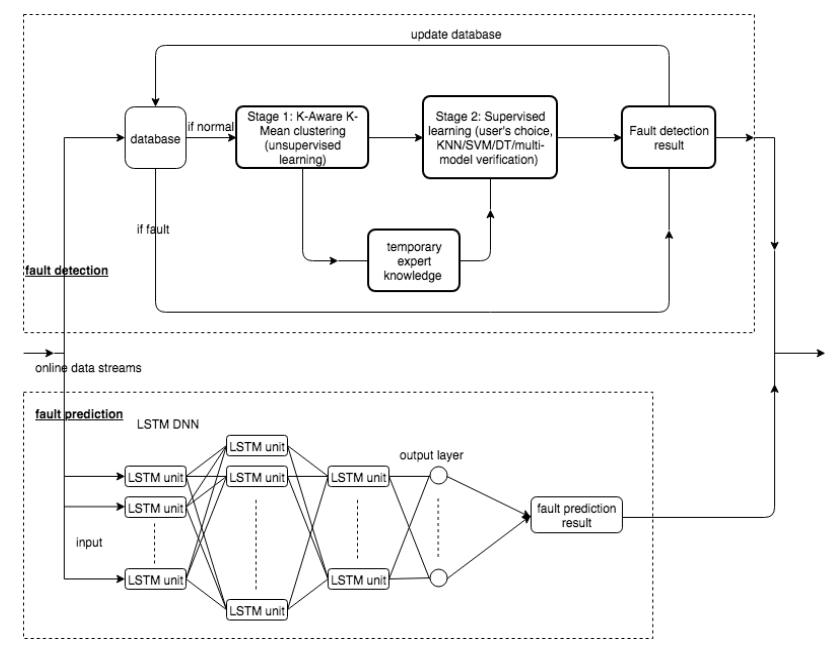

Figure 3. Fault Detection and Prediction Model

\section{MACHINE LEARNING}

In the machine learning model, both fault detection and prediction applications are embedded for sophisticated fault handling, which is described in Fig. 3. The machine learning model is software defined and takes in online data streams inputs to feed them to both (or either) the fault detection and fault prediction sub-models.

In the fault detection, a batch of online data streams are checked by the database as an initial examination for faults to see if they are recognized to have faults by the expert knowledge which keeps a record of existing fault types. If the data streams pass the initial check, then a combination of unsupervised and supervised learning algorithms are performed on the data for fault classification. The unsupervised learning algorithm, named as K-Aware KMean, is an extension of the conventional K-Mean clustering algorithm with additional capacity to self-optimize the $K$ value $(K \leq 1)$. This unsupervised learning phase aims to acquire a temporary expert knowledge of what the minority of the current data (the smallest cluster) is like and labels them as outliers; subsequently, a choice of the classical supervised learning algorithm is conducted based on the temporary expert knowledge to classify new types of faults comparing the database expert knowledge. Once a new type of fault is validated by the system, the database expert knowledge is renewed and updated.

In the fault prediction sub-model, a long short-term memory (LSTM) deep neural network (DNN) is proposed to perform online fault prediction. A LSTM netowrk is known as a popular recurrent neural network (RNN) for time-series analysis. A deep structure of LSTM can potentially have high accuracy prediction when the size of data is large enough. A single LSTM unit has its own structure which is shown in Fig.4. $\mathbf{x} \in \mathbb{R}^{n}$ and $\mathbf{h} \in \mathbb{R}^{h}$ are the input and the first hidden layer output of the LSTM-DNN. $t$ denotes the time step. $\mathbf{i}, \mathbf{o}$ and $\mathbf{f}$ are the input gate, output gate and forget gate vectors, respectively. $\mathbf{c} \in \mathbb{R}^{h}$ is the cell state vector. The hyperparameters include the weight matrices $\mathbf{W}_{i}, \mathbf{U}_{i}, \mathbf{W}_{o}$, 


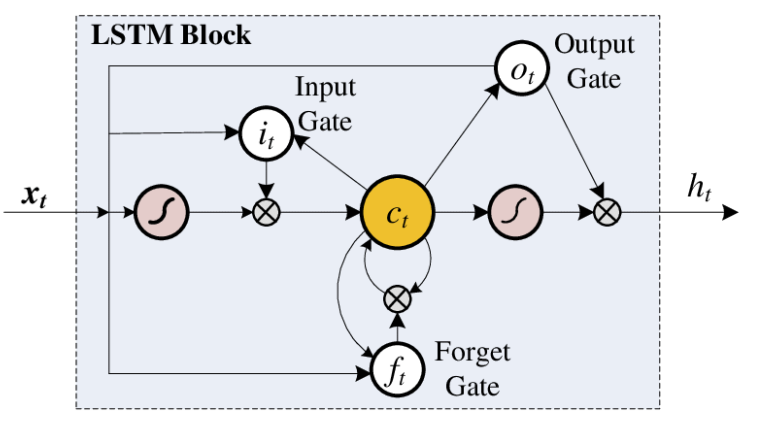

Figure 4. A single LSTM unit block diagram

$\mathbf{U}_{o}, \mathbf{W}_{f}, \mathbf{U}_{f}, \mathbf{W}_{c}$ and $\mathbf{U}_{c}$, biases $\mathbf{b}_{i}, \mathbf{b}_{o}, \mathbf{b}_{f}$ and $\mathbf{b}_{c}$, the activation functions $\sigma_{g}, \sigma_{h}$ and $\sigma_{c}$. The following equations describe their relationships:

$$
\begin{gathered}
\mathbf{i}_{t}=\sigma_{g}\left(\mathbf{W}_{i} \mathbf{x}_{t}+\mathbf{U}_{i} \mathbf{h}_{t}+\mathbf{b}_{i}\right), \\
\mathbf{o}_{t}=\sigma_{g}\left(\mathbf{W}_{o} \mathbf{x}_{t}+\mathbf{U}_{o} \mathbf{h}_{t}+\mathbf{b}_{o}\right), \\
\mathbf{f}_{t}=\sigma_{g}\left(\mathbf{W}_{f} \mathbf{x}_{t}+\mathbf{U}_{f} \mathbf{h}_{t}+\mathbf{b}_{f}\right), \\
\mathbf{c}_{t}=\mathbf{f}_{t} \circ \mathbf{c}_{t-1}+\mathbf{i} \circ \sigma_{c} \mathbf{W}_{c} \mathbf{x}_{t}+\mathbf{U}_{c} \mathbf{h}_{t}+\mathbf{b}_{c}, \\
\mathbf{h}_{t}=\mathbf{o}_{t} \circ \sigma_{h}\left(\mathbf{c}_{t}\right),
\end{gathered}
$$

where $\circ$ denotes the Hadamard product.

\section{INDUSTRIAL PROCESS AND Robots}

\section{A. Industrial Process}

The application of the proposed system architecture is investigated on a mini plant (see Fig. 5), available at the Department of Chemical and Process Engineering, University of Surrey (Guildford, United Kingdom). The plant produces sodium ion solution for sale to fine chemical, pharmaceutical and food industry. The raw material (sodium chloride contaminated with calcium chloride) is pre-mixed with sodium bicarbonate. This feedstock is then fed into a reactor vessel charged with pre-heated water, and reacted at $65 \mathrm{C}$. The suspension resulted from the reaction is pumped by positive displacement pump through a plate filter, which removes a high proportion of the calcium carbonate. The output stream splits into a product and a recycle line. The recycle is fed back to the reactor, while the product is passed through a polishing filter, to remove any remaining solids, and then to the lot tank. The carbon dioxide resulted in the reaction is absorbed in an alkaline solution. The plant is provided with sensors that monitor forty (40) process variables (e.g. temperature, pressure, processing unit levels) and a distributed control system that uses a cooperative model predictive control approach. The sensors are transmitting data every second.

\section{B. Robots}

The inspection of industrial sites, structures, and equipment are important issues for their sustainability. Although these tasks are repetitive and time-consuming, and some of these environments may be hazardous, it is still relied on humans for doing these operations. Robots are useful for

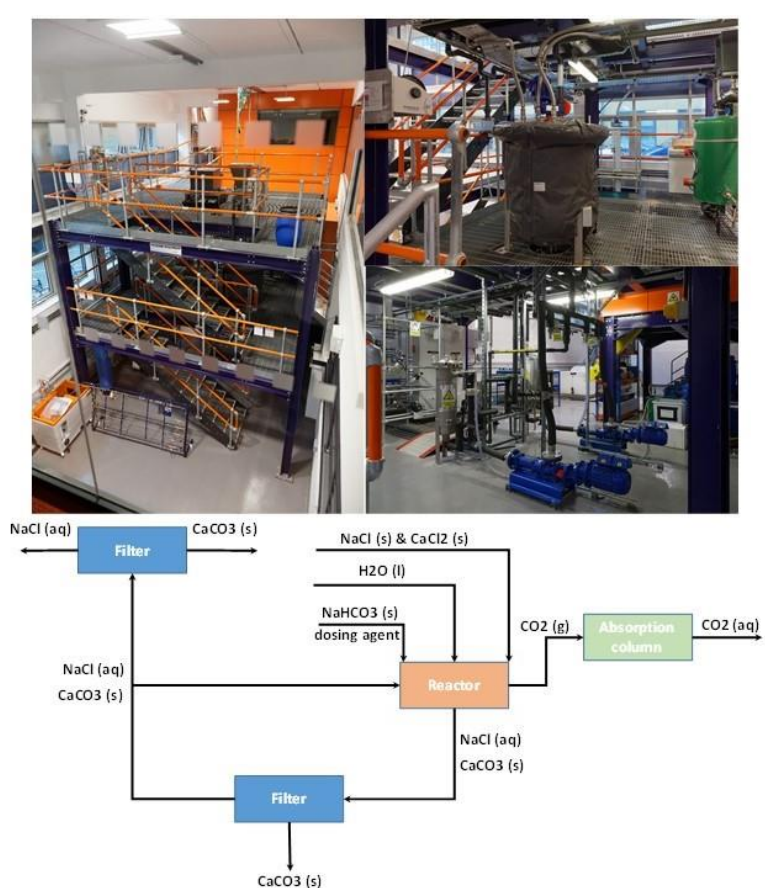

Figure 5. Mini Plant and Simplified Process Diagram

these tasks as they can be effective in exploring dangerous or inaccessible sites, at relatively low-cost and reducing the required time for executing them. Another reason is the better use of qualified professionals' capabilities for other jobs than routine patrolling. As modern plants become larger and more complex, the current robot's capabilities enable the achievement of these functions efficiently [11]. The mobile robots perform routine inspection and early fault detection in large process plants will provide fast and extensive coverage of large or hard-to-access plant structures by employing noncontact sensors. Among the regular inspection tasks that could be executed by the robots it can be mentioned the monitoring of gauges and meters, the inspection of valves, acoustic inspection, and inspection for leakage [12].

In the proposed model, the mobile robots will autonomously patrol around the plant. They are equipped with sensors for recording the necessary measurements, performing the periodic inspection and surveillance, as well as acting as relays in deep fading areas. The mobile robot exploited in this system is shown in Fig. 6. The robust robot navigation is vital for these applications, as the robot needs to make a frequent loop closure to achieve the tasks. During this work, the robot navigation has been done using the standard robot operating system navigation stack.

\section{RESUlTS}

This section shows simulation results in terms of sensor measurement fault detection and prediction applications of our $6 \mathrm{~S}$ system. In addition, it provides the OMA/NOMA boundary within the considered model. The dataset used for simulations was obtained from the Fluor pilot plant, 


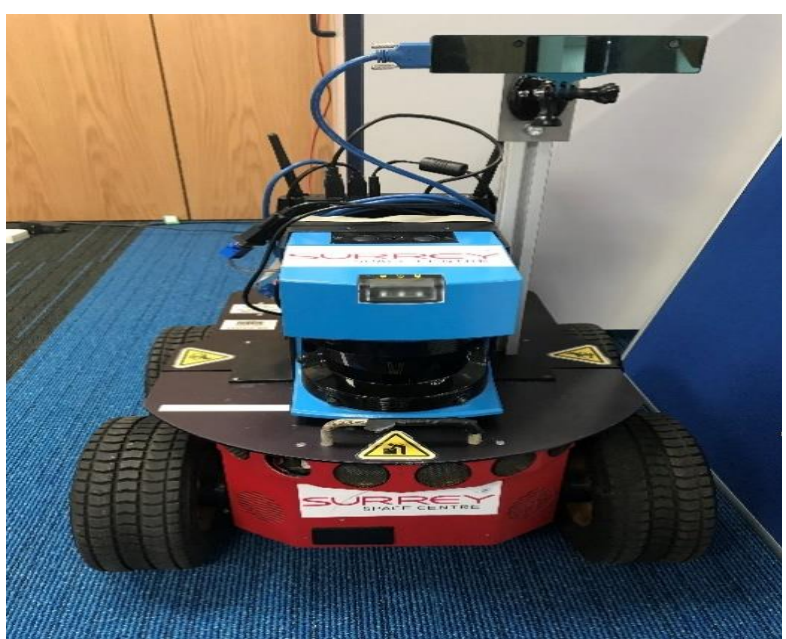

Figure 6. Inspector mobile robot equipped with laser range finder for navigation and RGB-D camera for visual inspection

Table I

COMPLEXITY COMPARISONS

\begin{tabular}{|c|c|}
\hline supervised learning classifier & detection accuracy \\
logistic regression & $97.5 \%$ \\
KNN & $100.0 \%$ \\
DT & $99.4 \%$ \\
linear SVM & $99.5 \%$ \\
\hline
\end{tabular}

University of Surrey, UK, in year 2017-2018. The data set has over $10 \mathrm{M}$ samples and each sample contains measurements for 43 variables. This variables include temperatures, flows, pressures and levels, and are collected by the deployed wireless sensors set up on the operational process units. The data samples were collected at a frequency of $1 \mathrm{~Hz}$ (i.e. one sample per second). Data types include both floating point and Boolean value data. The batch size for both fault detection and prediction algorithms is set as $10 \mathrm{~K}$. All simulations are conducted in the Python software and the Keras application programming interface (API) with Tensorflow backend. The computer hardware used comprises an Intel i7-8700 CPU (6Core/12-Thread, 12MB Cache, up to $4.6 \mathrm{GHz}$ with Intel Turbo Boost Technology) and dual NVidia GeForce GTX 1080 Ti GPUs (with 11GB GDDR5X each). For all supervised learning algorithms, the train-test split ratio is chosen at $(0.75,0.25)$. During the fault detection, for visualization purposes, the result of classifying the original high dimensional data is projected into a lower dimension (2D plane) using principal component analysis (PCA) as shown in Fig.7. Table I compares different supervised learning algorithms regarding fault detection accuracy.

For fault prediction, a three hidden layer LSTM-DNN is configured to perform one time step ahead prediction for 40 dynamic variables. Note that the proposed DNN performs more than just an auto-regressor, as the fault decision criteria is based on the tracking of a threshold that determines how well the predicted values at next time step are generally matching the actual values at the current time step, but for a

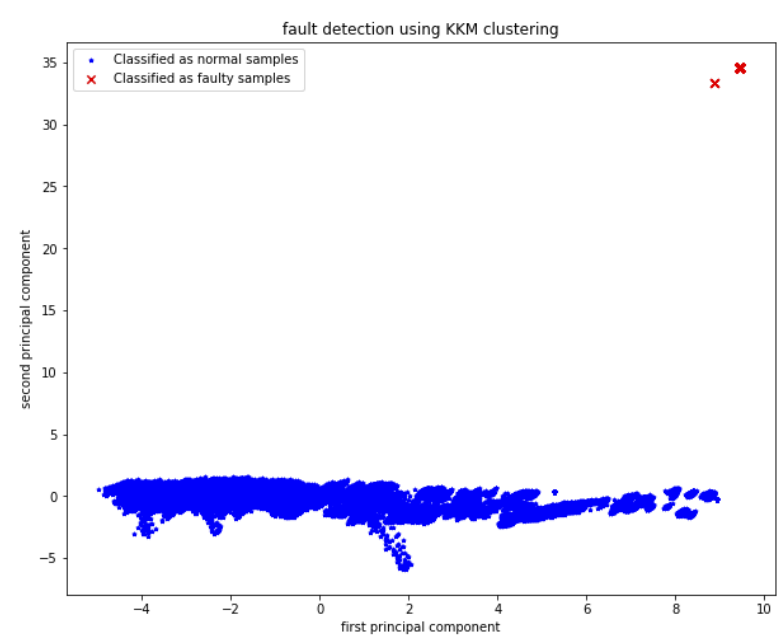

Figure 7. A single LSTM unit block diagram

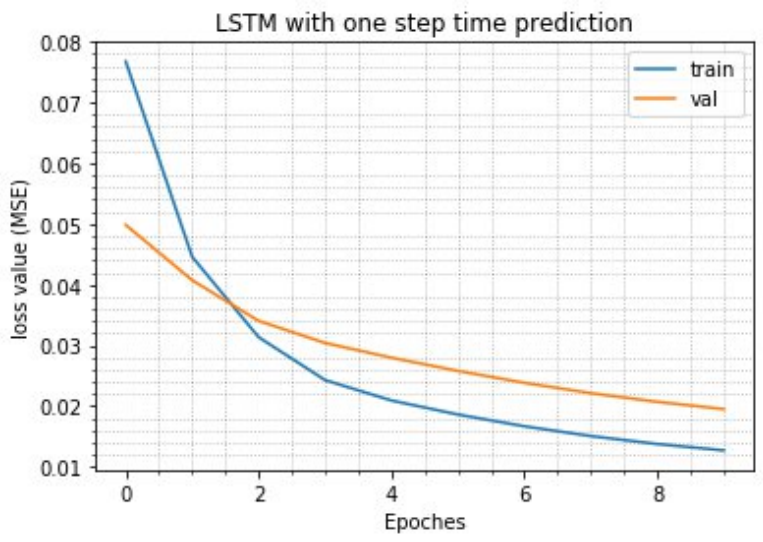

Figure 8. A single LSTM unit block diagram

consecutive number of time steps. The dataset train-test ratio is still set at $(0.75,0.25)$. For each of the hidden layers, 30, 50 , and 30 LSTM units are used, respectively. The mini-batch method is used to accelerate the stochastic gradient optimizer (step size is set as 0.1 , to minimize the mean squared error, i.e. MSE between the predicted values and actual values of the variables) based training in every training epoch. Each LSTM hidden layer uses a linear activation function and the same applies in the output layer. 3 human-controlled variables named as "Connection", "Alarms" and "E-Stop" are excluded from the dataset to reduce undesirable human factor effects. The proposed LSTM-DNN is able to achieve a prediction accuracy of $99.7 \%$ according to the results. The train-test learning curve comparison is illustrated in Fig. 8.

The boundary between OMA and NOMA is investigated for both URLLC and mMTC use cases. In the former, each sensor is allocated resources to achieve at least the required data rate to ensure low latency and high reliability, while in the mMTC the resources are distributed among larger number of sensors to ensure an average data rate for each sensor. The frame structure we proposed in [13] is considered. A 400 $\mathrm{kHz}$ bandwidth is divided equally between the URLLC and 


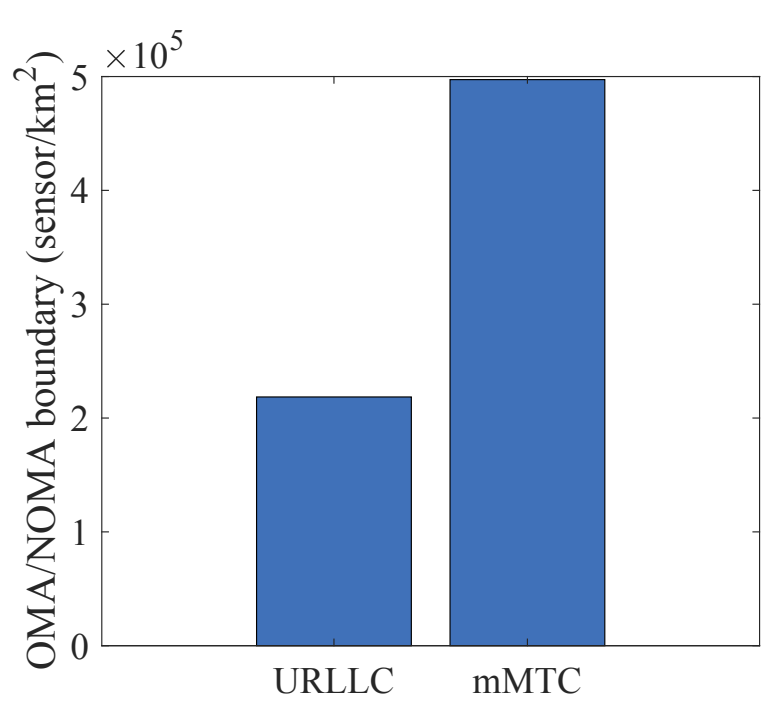

Figure 9. Maximum sensor density with OMA

the mMTC slices (i.e., $200 \mathrm{kHz}$ each). Each sensor generates 125 bytes packets and the Poisson traffic model is considered. The reader is referred to [13] for details of the frame structure and transmission models.

Fig. 9 shows the OMA/NOMA boundary for both use cases. As can be seen, a maximum density of 218500 sensors $/ \mathrm{km}^{2}$ can be supported for URLLC with OMA. Any higher density will require a NOMA-based tranmission. On the other hand, the mMTC use case allows a higher density of 497300 sensors $/ \mathrm{km}^{2}$ with OMA. This can be traced to the fact mMTC allows relaxed latency requirements to accommodate more sensors as opposed to the tight latency constraints of the URLLC. Hence it can be said that the network dimensioning and sensor density in industrial plants is highly influenced by the underlying requirements. This suggests a sliced-based allocation for verticals of $5 \mathrm{G}$ networks.

\section{CONCLUSION}

In this paper, we developed a high level inter-disciplinary modelling approach leveraging capabilities of wireless sensors in $5 \mathrm{G}$ networks and machine learning algorithms to step towards the $6 \mathrm{~S}$. Since different verticals/applications have different requirements/elements, the proposed approach is built in a modular manner. Focusing on industrial processes and chemical plants as a case study, we adapted the proposed model to control elements of the plant. The latter includes physical controls, sensors and actuators as well as inspection robots overlaid by a wireless network. A higher decision layer is proposed for fault detection and prediction to optimise the plant operation. In addition, the appropriate sensor density based on the multiplexing scheme is investigated. The presented results indicate that proposed model achieve very high fault detection/prediction accuracy based on real plant measurements. The future work will consider the impact of mobile wireless sensors deployed in robots, as well as integrating the machine-learning based fault detection/prediction scheme with a plant/vertical-specific process models to achieve both network and process fault prediction.

\section{ACKNOWLEDGEMENT}

This work is supported by the U.K. Engineering and Physical Sciences Research Council (EPSRC) under Grant EP/R001588/1 for the project Stepping Towards the Industrial Sixth Sense. We would like to acknowledge the support of the University of Surrey 5GIC (http://www.surrey.ac.uk/5gic) members for this work.

\section{REFERENCES}

[1] M. A. Alsheikh, S. Lin, D. Niyato, and H.-P. Tan, "Machine learning in wireless sensor networks algorithms, strategies, and applications," IEEE Communication Surveys \& Tutorials, vol. 16, no. 4, 2014.

[2] L. S. Z. H. Y. Z. Z. Zhang, A. Mehmood and M. Mukherjee, "A survey on fault diagnosis in wireless sensor networks," IEEE Access, Feb 2018.

[3] J. Kwak, T. Lee, and C. O. Kim, "An incremental clustering-based fault detection algorithm for class-imbalanced process data," IEEE Transactions on Semiconductor Manufacturing, vol. 28, no. 3, pp. 318 - 328, June 2015.

[4] S. Rashid, U. Akram, S. Qaisar, S. A. Khan, and E. Felemban, "Wireless sensor network for distributed event detection based on machine learning," in 2014 IEEE International Conference on Internet of Things (iThings), and IEEE Green Computing and Communications (GreenCom) and IEEE Cyber, Physical and Social Computing (CPSCom), Sep 2014, pp. 540 - 545.

[5] H. Martins, F. Januário, L. Palma, A. Cardoso, and P. Gil, "A machine learning technique in a multi-agent framework for online outliers detection in wireless sensor networks," in IECON 2015 - 41st Annual Conference of the IEEE Industrial Electronics Society, Nov 2015, pp. $000688-000693$.

[6] S. Zidi, T. Moulahi, and B. Alaya, "Fault detection in wireless sensor networks through svm classifier," IEEE Sensors Journal, vol. 8, no. 1, pp. $340-347$, Nov 2018.

[7] M. Parwez, D. B. Rawat, and M. Garuba, "Big data analytics for user-activity analysis and user-anomaly detection in mobile wireless network," IEEE Transactions on Industrial Informatics, vol. 13, no. 4, pp. 2058 - 2065, Aug 2017.

[8] A. C. Onal, O. B. Sezer, M. Ozbayoglu, and E. Dogdu, "Mis-iot: Modular intelligent server based internet of things framework with big data and machine learning," in 2018 IEEE International Conference on Big Data (Big Data), Dec 2018, pp. 2270 - 2279.

[9] Z. Gao, L. Ma, and J. Wang, "Fault tolerant control method for displacement sensor fault of wheel-legged robot based on deep learning," in 2018 WRC Symposium on Advanced Robotics and Automation (WRC SARA), Aug 2018, pp. 147 - 152.

[10] F. Calivá, F. S. D. Ribeiro, A. Mylonakis, C. Demazi'ere, P. Vinai, G. Leontidis, and S. Kollias, "A deep learning approach to anomaly detection in nuclear reactors," in 2018 International Joint Conference on Neural Networks (IJCNN), July 2018, pp. 1 - 8.

[11] P. Kohlhepp and G. Bretthauer, "Cooperative service robots for the predictive maintenance of process plants," in Proc. Int. Colloq. on Autonomous and Mobile Systems, Magdeburg, GERMANY, vol. 25. Citeseer, 2002.

[12] M. Bengel, K. Pfeiffer, B. Graf, A. Bubeck, and A. Verl, "Mobile robots for offshore inspection and manipulation," in IEEE/RSJ International Conference on Intelligent Robots and Systems, 2009, pp. 3317-3322.

[13] A. Ijaz, L. Zhang, M. Grau, A. Mohamed, S. Vural, A. U. Quddus, M. A. Imran, C. H. Foh, and R. Tafazolli, "Enabling massive iot in $5 \mathrm{~g}$ and beyond systems: Phy radio frame design considerations," IEEE Access, vol. 4, pp. 3322-3339, 2016. 